\title{
Dose accuracy improvement on head and neck VMAT treatments by using the Acuros algorithm and accurate FFF beam calibration
}

\author{
Guadalupe Martin-Martin ${ }^{1}$, Stefan Walter ${ }^{2}$ Eduardo Guibelalde ${ }^{3}$ \\ ${ }^{1}$ Medical Physics and Radiation Protection Service, Hospital Universitario de Fuenlabrada, Madrid, Spain \\ ${ }^{2}$ Department of Medicine and Public Health, Rey Juan Carlos University, Alcorcón, Spain \\ ${ }^{3}$ Medical Physics Group, Department of Radiology, University Complutense of Madrid, Madrid, Spain
}

\begin{abstract}
Background: The purpose of this study was to assess dose accuracy improvement and dosimetric impact of switching from the anisotropic analytical algorithm (AAA) to the Acuros XB algorithm (AXB) when performing an accurate beam calibration in head and neck (H\&N) FFF-VMAT treatments.

Materials and methods: Twenty H\&N cancer patients treated with FFF-VMAT techniques were included. Calculations were performed with the $A A A$ and $A X B$ algorithm (dose-to-water $-\mathrm{AXB}_{w}$ - and dose-to-medium - $A X B_{m}-$ ). An accurate beam calibration was used for AXB calculations. Dose prescription to the tumour (PTV70) and at-risk-nodal region (PTV58.1) were 70 Gy and 58.1 Gy, respectively. A PTV70 _bone including bony structures in PTV70 was contoured. Dose-volume parameters were compared between the algorithms. Statistical tests were used to analyze the differences in mean values and the correlation between compliance with the D95 > 95\% requirement and occurrence of local recurrence.

Results: AAA systematically overestimated the dose compared to AXB algorithm with mean dose differences within 1.3 $\mathrm{Gy} / 2 \%$, except for the PTV70 _bone $(2.2 \mathrm{~Gy} / 3.2 \%)$. Dose differences were significantly higher for $\mathrm{AXB}_{\mathrm{m}}$ calculations when including accurate beam calibration (maximum dose differences up to $2.8 \mathrm{~Gy} / 4.1 \%$ and $4.2 \mathrm{~Gy} / 6.3 \%$ for PTV70 and PTV70 bone, $_{\text {respec- }}$ tively). $80 \%$ of AAA-calculated plans did not meet the D95 > 95\% requirement after recalculation with $\mathrm{AXB}_{\mathrm{m}}$ and accurate beam calibration. The reduction in D95 coverage in the tumour was not clinically relevant.

Conclusions: Using the $\mathrm{AXB}_{\mathrm{m}}$ algorithm and carefully reviewing the beam calibration procedure in H\&N FFF-VMAT treatments ensures (1) dose accuracy increase by approximately 3\%; (2) a consequent dose increase in targets; and (3) a dose reporting mode that is consistent with the trend of current algorithms.
\end{abstract}

Key words: Acuros XB (AXB); Anisotropic Analytical Algorithm (AAA); FFF-VMAT; head and neck (H\&N)

Rep Pract Oncol Radiother 2021;26(1):73-85

\section{Introduction}

The recent trend in radiotherapy has been to treat head and neck $(\mathrm{H} \& \mathrm{~N})$ cancer patients via intensitymodulated radiation therapy (IMRT) techniques. Treatments of this type are being replaced by volu- metric modulated arc therapy (VMAT) techniques which are faster and, consequently, more favourable to the patient. In addition, for these techniques, the use of flattening filter free (FFF) beams is a superior choice, as the treatment time can be reduced by a factor of 2-4 [1]. While this reduction in treatment

Address for correspondence: Guadalupe Martin-Martin, Hospital Universitario de Fuenlabrada, c/Camino del Molino, 2, 28942 Fuenlabrada, Madrid, Spain; e-mail: guadalupe.martin@salud.madrid.org

This article is available in open access under Creative Common Attribution-Non-Commercial-No Derivatives 4.0 International (CC BY-NC-ND 4.0) license, allowing to download articles and share them with others as long as they credit the authors and the publisher, but without permission to change them in any way or use them commercially 
time associated with FFF beams was initially found to be particularly useful for small field stereotactic radiosurgery (SRS) and/or stereotactic body radiotherapy (SBRT) procedures [2], it was later found to be useful for a wide range of fields and treatments as well $[3,4]$. However, the unique characteristics of unfiltered beams, which provide much higher dose rates than conventional beams, may impact the accuracy of the dose determination in radiotherapy treatments that use such beams $[1,3]$.

Additionally, dose calculation in areas of various densities, such as bone, soft tissue, cartilage and air in head and neck $(\mathrm{H} \& \mathrm{~N})$ cancer patients, poses a challenge for dose calculation algorithms. Therefore, in addition to the uncertainties that are associated with FFF beam absolute dose calibration, errors in the overall dose determination in these treatments may also originate from inaccuracies in the dose determination by treatment planning system (TPS) algorithms [5, 6].

The dose calculation algorithms implemented in TPS can be divided into three categories [7,8], based on their degree of complexity in the management of charged particle transport. Type "a" algorithms are usually based on pencil beam convolution principles; more advanced type "b" algorithms, based on superposition-convolution principles, provide increased accuracy over type "a" algorithms, especially in the presence of tissue heterogeneities. However, type "b" algorithms, are of limited accuracy in dose distribution calculation in heterogenous areas, and the error can reach $20 \%$ in extreme cases [5]. Type "c" algorithms, are grid-based linear Boltzmann transport equation solvers and represent the most recent class of dose calculation algorithms with increased accuracy over type " $b$ " algorithms.

The shortcomings of the anisotropic analytic algorithm (AAA), a type "b" algorithm that is commonly used in current clinical practice [9], are well documented [10-14]. For treatments of areas of high heterogeneity, such as H\&N treatments, Robinson [14] showed that the AAA algorithm is unable to correctly handle altered attenuation along large density gradients. To address these shortcomings, the Acuros XB (AXB) algorithm, which is a type "c" algorithm, was implemented in the Eclipse planning system (Varian Medical Systems, Inc., Palo Alto, CA, USA) in 2010 [15]. Since then, several studies $[10,16-20]$ have shown that the accuracy of the AXB algorithm in heterogeneous media is com- parable to that of the Monte Carlo methods, which are considered the most accurate dose calculation methods in radiotherapy [21]. All this has raised awareness of the need to accurately determine the dose distribution in current $\mathrm{H} \& \mathrm{~N}$ treatments [12, 22-29]. Consequently, several authors [12, 23-28] have shown that the AAA algorithm systematically overestimates the dose that is delivered to the tumour compared to the AXB algorithm.

However, to the best of our knowledge, there are no studies published which have evaluated the performance of the Acuros algorithm against AAA in H\&N VMAT treatments that are produced by FFF beams. In this context, we conducted a retrospective study with $\mathrm{H} \& \mathrm{~N}$ patients who were previously treated in our centre via the FFF-VMAT technique and the AAA algorithm was used to compute the treatment plans. These plans were compared with the Acuros recalculated plans with the two reporting modes of AXB (dose-to-water and dose-to-medium), which included a more robust calibration of the unfiltered beam than recommended in the IAEA/AAPM TRS483 [30] and AAPM TG-51 addendum [31] protocols for reference dosimetry. This calibration mainly consisted in considering the significant impact of the ion recombination effect on relative dosimetry of FFF beams for certain chamber models [32-35], which may affect the absolute dose calibration [32].

The objective of this study was, therefore, to assess dose accuracy increase and dosimetric impact of switching from the AAA to the AXB algorithm in H\&N FFF-VMAT treatments when performing an accurate FFF beam calibration. Furthermore, based on the results of this work, we aimed to determine whether it was essential for us to switch to one of the two modes of the Acuros algorithm in our H\&N treatments and to perform such characterization of the unfiltered beam.

\section{Materials and methods}

\section{Ion chamber measurements}

The ion chambers that were used in this study were: a Farmer-type chamber (PTW 30013 with SN 003265), a small-volume Semiflex 3D-type chamber (PTW 31021 with SN 142013) and a scanning chamber (IBA CC13 with SN 15496), with nominal volumes of $0.6,0.07$ and $0.13 \mathrm{~cm}^{3}$, respectively.

All measurements were conducted on a Siemens Artiste linear accelerator (Siemens AG, Erlangen, 
Germany) using an unflattened X-ray beam with a nominal energy of 7 MV UF (hereinafter 7XFFF, with a measured dose per pulse of $1.5 \mathrm{mGy} /$ pulse at the depth of the maximum dose, namely, $\mathrm{d}_{\max }$, and a nominal dose rate of $2000 \mathrm{MU} / \mathrm{min}$ ). Ion chamber measurements were conducted in a water phantom (MP1, PTW-Freiburg) of dimensions $32 \mathrm{~cm} \times 32$ $\mathrm{cm} \times 37 \mathrm{~cm}$, with a $10 \times 10 \mathrm{~cm}^{2}$ field for a $100 \mathrm{~cm}$ source-to-surface distance (SSD) setup. Variations in the accelerator output were considered using an external monitor chamber, which was positioned in water, within the field, at a measurement depth of approximately $4 \mathrm{~cm}$ lateral to the chamber that was being tested, as recommended in the IAEA TRS-398 Code of Practice [30].

\section{FFF beam calibration method}

We investigated the dosimetric errors in the absolute dose calibration of the unflattened beam that were due to two main effects: (1) ion recombination effects that were due to the higher dose per pulse in FFF beams and (2) volume-averaging effects for the peaked profile of the unflattened beam.

To assess the error in the measurements that was due to ion recombination effects in the unflattened beam, the impact of this effect on the percentage depth dose (PDD) curves was investigated. The accuracy of the measurement of percentage depth dose at $10 \mathrm{~cm}$ depth $(\mathrm{PDD}(10))$ was assessed, as this value is relevant for the determination of the absorbed dose to water at dose maximum according to the TRS-398 protocol for absolute dosimetry.

The relative error between the uncorrected $\operatorname{PDD}(10)_{u}$ and the corrected $\operatorname{PDD}(10)_{c}$ was calculated as follows [33]:

$$
\Delta_{\% P D D(10)}=100 \frac{\left(P D D(10)_{c}-P D D(10)_{u}\right)}{P D D(10)_{c}}
$$

Furthermore, the relative error in the measurements for the determination of the absorbed dose to water that was due to volume-averaging effects in the unflattened beam was calculated as follows [32]:

$$
\Delta_{\% v o l}=100 \times\left(1-P_{r p}\right)
$$

Where $\mathrm{P}_{\mathrm{rp}}$ accounts for any off-axis variation in the intensity profile of the radiation field over the sensitive volume of the ionization chamber [31].
Additional details on the measurements and formulation required to obtain equations (1) and (2) are provided in Appendix A.

\section{Patient selection and prescription}

This study was conducted under the approval of the Committee for Ethical Research (19/32) of the hospital. All patient data that were used in this study were anonymized. Twenty patients who were diagnosed with $\mathrm{H} \& \mathrm{~N}$ cancer and were treated with the FFF-VMAT technique with concomitant chemotherapy in our institution, from September 2016 to September 2017, were reviewed. The average age of the patients was 63 years old (52-78), with $80 \%$ men and $20 \%$ women. Additional patient characteristics are provided in Supplementary Table 1. CT scanning was conducted using a Siemens Somatom CT scanner (Siemens AG, Erlangen, Germany), and all patients were immobilized with a thermoplastic head and shoulder mask.

Accelerated radiotherapy with simultaneous integrated boost (SIB) fractionation was delivered in 35 fractions using the VMAT technique with the 7XFFF beam. The total doses that were prescribed to the planning target volumes (PTVs) were 70 Gy and 58.1 Gy with daily doses of 2 Gy and 1.66 Gy, respectively. PTV70 and PTV58.1, which correspond to the prescribed doses of 70 and $58.1 \mathrm{~Gy}$, respectively, were delineated by the radiation oncologist, and a 0.5 $\mathrm{cm}$ isotropic margin was applied to their applicable clinical target volumes (CTV). CTV70 for PTV70 included gross tumour, and CTV58.1 for PTV58.1 included at-risk nodal regions. In addition, to assess the dosimetric impact to PTVs due to materials of different densities, a new PTV70_bone was contoured which included bony structures in PTV70. We did not assess similarly a PTV58.1_bone because of the low proportion of bone that was found in at-risk nodal regions. The dosimetric impact to PTVs due to materials of different densities other than bone was not evaluated either, as previous studies proved that only bony structures impacted dose differences when comparing Acuros with type $b$ algorithms in $H \& N$ cancer [24-26]. The brainstem, spinal cord, larynx, brain, cochleas, parotid glands, thyroid gland and mandible were delineated as organs at risk (OARs). 


\section{Planning and dose calculation}

Treatment plans were created using the Eclipse TPS (version 15.5, Varian Medical System, Palo Alto, CA, USA). The plans were generated with the 7XFFF beam using a two-full-arc, mArc technique, which is the Siemens solution for VMAT treatments. All plans were optimized until they covered at least $95 \%$ of the two PTVs with $95 \%$ of the prescribed dose (D95 $\geq 95 \%)$. In addition, a plan was clinically acceptable if the maximum dose to both PTVs was $\leq 107 \%$ of the prescribed dose and the dose-volume constraints for OAR met the following criteria: maximum dose to the spinal cord and thyroid gland < $45 \mathrm{~Gy}$; maximum dose to the mandible and larynx < 70 Gy; maximum dose to the brainstem and Cochleas < 53 Gy and $50 \mathrm{~Gy}$, respectively; volume of brainstem receiving $60 \mathrm{~Gy}$ $\leq 30 \%$; and volume of parotid glands receiving 30 Gy $\leq 50 \%$ with mean doses $\leq 26$ Gy.

All H\&N mArc plans were calculated using the AAA algorithm and recalculated using the Acuros $\mathrm{XB}$ algorithm for the dose-to-medium $\left(\mathrm{AXB}_{\mathrm{m}}\right)$ and dose-to-water $\left(\mathrm{AXB}_{\mathrm{w}}\right)$ reporting modes and the same number of monitor units (MU). A grid size of $2.5 \mathrm{~mm}$ was adopted for all plans. A new dose per MU factor, which included the corrections for the absolute dose calibration of the FFF beam, was introduced into the TPS to conduct the AXB calculations. $\mathrm{AXB}_{\mathrm{w}, \mathrm{FFF}}$ and $\mathrm{AXB}_{\mathrm{m}, \mathrm{FFF}}$ represent the recalculated plans that include these corrections.

\section{Dosimetric parameters}

The Eclipse Visual Scripting v15.1 tool was used to obtain the desired dosimetric parameters for the PTVs - this study does not focus on the OAR doses - for all patients for each calculation mode $\left(\mathrm{AAA}, \mathrm{AXB}_{\mathrm{m}}, \mathrm{AXB}_{\mathrm{w}}, \mathrm{AXB}_{\mathrm{w}, \mathrm{FFF}}\right.$ and $\mathrm{AXB}_{\mathrm{m}, \mathrm{FFF}}$ ).

According to the recommendations of ICRU 83, the following PTV dose-volumetric parameters were obtained for all plans: near minimum absorbed dose D98 and D95, median absorbed dose D50, near maximum absorbed dose D2 and the homogeneity index $(\mathrm{HI}=(\mathrm{D} 2-\mathrm{D} 98) / \mathrm{D} 50)$ for PTV70.

\section{Data analysis of AAA versus $A X B_{m}$ and $A X B_{w}$ modes}

The mean value and standard deviation (SD) of every dose-volumetric parameter for all PTVs were calculated for each calculation mode. To investigate the statistical significance of the differences in the mean values of each dose-volume parameter that were calculated with $\mathrm{AAA}$ versus $\mathrm{AXB}_{\mathrm{m}}$ and $\mathrm{AXB}_{w}$, p-values were calculated using the student's t-test with $95 \%$ confidence intervals. The statistical analysis was conducted using the $\mathrm{R}$ software, version 3.5.3 (R: A language and environment for statistical computing. R foundation for Statistical Computing, Vienna, Austria). The test determined whether the difference in the means for each considered dose metric was equal to zero. Additionally, the Fisher exact test was also used to investigate the statistical significance between complying with the requirement of D95 $\geq 95 \%$ of the prescribed dose for the tumour and the occurrence of local recurrence. A statistically significant $\mathrm{p}$-value indicates that not achieving the stipulated minimum dosage of the D95 $\geq 95 \%$ requirement was related to the occurrence of local recurrence for a given calculation mode $\left(\mathrm{AXB}_{\mathrm{m}}\right.$ or $\left.\mathrm{AXB}_{\mathrm{w}}\right)$. For both cases, values of $\mathrm{p}<0.05$ were considered statistically significant.

In addition, the relative dose differences between the dose-volumetric parameters of the $\mathrm{AXB}_{\mathrm{m}}$ or $\mathrm{AXB}_{\mathrm{w}}$-based plans and the AAA-based plans for each patient case were calculated via the following equation:

$$
\text { Rel. dose diff. from } A A A(\%)=\frac{\left(A X B_{y, x}-A A A_{x}\right)}{A A A_{x}} 100
$$

where $y$ correlates with the selected calculation mode for $\mathrm{AXB}\left(\mathrm{AXB}_{\mathrm{m}}\right.$ or $\left.\mathrm{AXB}_{\mathrm{w}}\right)$ and $\mathrm{x}$ is the corresponding dose-volumetric parameter (D98, D95, D50 or D2). The mean value and the standard deviation of the relative dose differences for every dosimetric parameter were plotted and analysed.

$$
\begin{aligned}
& \text { Data analysis of } A A A \text { versus } A X B_{m, F F F} \\
& \text { and } A X B_{w, F F F} \text { modes }
\end{aligned}
$$

A data analysis similar to that of the previous section was conducted for the comparison between AAA and those AXB modes which included the corrections for the absolute dose calibration of the FFF beam $\left(\mathrm{AXB}_{\mathrm{m}, \mathrm{FFF}}\right.$ and $\left.\mathrm{AXB}_{\mathrm{w}, \mathrm{FFF}}\right)$

\section{Results}

\section{Systematic errors in the absolute output dosimetry of the FFF beam}

According to our measurements, the reduced ion collection efficiency influences the measured $\mathrm{PDD}(10)$. In addition, non-negligible volume-av- 
Table 1. Relative systematic errors as percentages for the uncorrected PDD(10), $\triangle \% \mathrm{PDD}(10)$, and the volume-averaging effect, $\Delta \%$ vol. The uncertainties are the propagated coefficients of variation for these measurements from the calculated values of $k_{s}$ and $P_{r p}($ Appendix $A)$. The largest values of the systematic errors are presented in bold

\begin{tabular}{|l|c|c|}
\hline Ion chamber model & $\Delta \%$ PDD(10) & $\Delta \%$ vol \\
\hline PTW 30013 Farmer & $-0.2 \pm 0.05$ & $-0.3 \pm 0.01$ \\
\hline PTW 31021 Semiflex & $-0.3 \pm 0.05$ & $0.0 \pm 0.01$ \\
\hline IBA CC13 Scanning & $-0.7 \pm 0.05$ & $0.0 \pm 0.01$ \\
\hline
\end{tabular}

eraging effects were observed for the Farmer-type chamber in the FFF beam. Table 1 presents the relative systematic errors that were due to these effects for the chambers that were used in this study; the largest errors for the uncorrected $\mathrm{PDD}(10)$ and the volume-averaging effect were obtained for the IBA CC13 ion chamber and Farmer PTW 30013 chamber, which showed a $\Delta_{\% \text { PDD(10) }}$ of $-0.7 \%$ and $\Delta_{\% \text { vol }}$ of $-0.3 \%$, respectively. Therefore, when the IBA CC13 ion chamber is used to measure the $\operatorname{PDD}(10)$ and the Farmer PTW 30013 is used for the absolute output dosimetry, the overall systematic error is $-1 \%$. We used this worst-case scenario to assess the largest dosimetric impact of the overall systematic error. The 'FFF' subscript in $\mathrm{AXB}_{\mathrm{w}, \mathrm{FFF}}$ and $\mathrm{AXB}_{\mathrm{m}, \mathrm{FFF}}$ indicates that this $-1 \%$ systematic error has been included in the TPS calculations.

\section{$A A A$ versus $A X B$ calculations}

The means and SDs of the PTV dose-volumetric parameters for the $\mathrm{AAA}, \mathrm{AXB}_{\mathrm{m}}, \mathrm{AXB}_{\mathrm{w}}, \mathrm{AXB}_{\mathrm{w}, \mathrm{FFF}}$ and $\mathrm{AXB}_{\mathrm{m}, \mathrm{FFF}}$ calculations for the $20 \mathrm{H} \& \mathrm{~N}$ cases are listed in Table 2. It also shows the statistical significance of the differences in the mean values of the PTV dose-volume parameters between the AAA and AXB calculations. The differences in the mean values of all dose-volumetric parameters of PTV70, PTV70 $_{\text {bone }}$ and PTV58.1 were significantly different from $0(\mathrm{p}<0.05)$, except for the near maximum absorbed dose D2 to PTV70 for AAA vs. $\mathrm{AXB}_{w}$ and D98, D95 and D50 to PTV70_bone for AAA vs. $\mathrm{AXB}_{\mathrm{w}, \mathrm{FFF}}$. Additionally, the AAA plans showed significantly lower $\mathrm{HI}$ values $(\mathrm{p}<0.05)$ compared to the two AXB modes for PTV70 and PTV70 had been previously reported $[25,27,28]$, indicating that AAA produced more homogeneous plans.

Figure 1 completes this information by graphically depicting the average differences and standard deviations in the PTV dose-volume parameters between the AAA and AXB calculations, which are computed via equation (5). The $2 \%$ maximum recommended uncertainty of the computed dose distributions [36] is presented in plots (a) and (b) of Figure 1.

\section{$A A A$ versus the $A X B_{m}$ and $A X B_{w}$ modes}

As expected, Figure 1 supports our previous results: for all plan dose comparisons, AAA statistically overestimated the dose to PTV, except for D2 (Fig. 1B), for which AAA underestimated the dose relative to $\mathrm{AXB}_{\mathrm{w}}$. All dose-volume parameters that were linked to a specified value of PTV70 and PTV58.1 coverage (D98, D95 and D50) were systematically lower for any AXB mode compared to AAA, particularly in PTV70 for the $\mathrm{AXB}_{\mathrm{m}}$ mode. The maximum dose differences in D98, D95 and D50 in PTV70 were $-3.1 \%,-2.9 \%$ and $-3 \%$, respectively, for AAA vs. $\mathrm{AXB}_{\mathrm{m}}$. For AAA vs. $\mathrm{AXB}_{w}$, these differences were $-2 \%,-1.7 \%$ and $-1.6 \%$, respectively. Nevertheless, the average dose differences between AAA and the AXB modes were all within the $2 \%$ threshold (Fig. 1A, B).

Furthermore, the reduction of doses predicted by $\mathrm{AXB}_{\mathrm{m}}$ in PTV70_bone was notably higher than that in PTV70 (Fig. 1A), while higher doses were found in PTV70_bone for $\mathrm{AXB}_{\mathrm{w}}$ (Fig. 1B). The maximum dose differences in D98, D95, D50 and D2 in PTV70_bone were $-5.2 \%,-5.4 \%,-4.3 \%$ and $-3.5 \%$, respectively, for AAA vs. $\mathrm{AXB}_{\mathrm{m}}$, whereas the maximum dose difference in D2 of PTV70_bone was 6.7\% for AAA vs. $\mathrm{AXB}_{\mathrm{w}}$.

\section{$A A A$ versus the $A X B_{m, F F}$ and $A X B_{w, F F}$ modes}

Figure $1(\mathrm{C}, \mathrm{D})$ shows the effect of considering the FFF beam systematic errors in the TPS calculations when switching from AAA to both AXB modes. AAA overestimated the dose-volume parameters in PTV70 and PTV58.1 more severely than when these errors were not considered. The maximum dose differences for D98, D95, D50 and D2 in PTV70 were $-4.1 \%,-3.9 \%,-4 \%$ and $-3.3 \%$, respectively, for $A A A$ vs $\mathrm{AXB}_{\mathrm{m}, \mathrm{FFF}}$. In addition, the $\mathrm{D} 2$ values in PTV70 that were calculated by $\mathrm{AXB}_{w}$ and were systematically higher than those that were calculated by AAA, were partially compensated for the systematic errors in the unflattened beam. As a result, the average $\mathrm{D} 2$ values that were calculated by $\mathrm{AXB}_{\mathrm{w}, \mathrm{FFF}}$ were smaller than those that 
Table 2. Means and standard deviations of dose-volumetric parameters of PTV over the whole sample. P-values for the comparisons of $A A A$ vs. $A X B_{m}, A A A$ vs. $A X B_{m}, F F F, A A A$ vs. $A X B_{w}$ and $A A A$ vs. AXB $B_{w}, F F F$. Statistically significant $p$-values are in bold

\begin{tabular}{|c|c|c|c|c|c|c|c|c|c|}
\hline & AAA & $\mathrm{AXB}_{\mathrm{m}, \mathrm{FFF}}$ & $\begin{array}{l}\text { AAA vs. } \\
\text { AXB }_{\mathrm{m}, \mathrm{FFF}}\end{array}$ & $\mathrm{AXB}_{\mathrm{m}}$ & $\begin{array}{c}\text { AAA vs. } \\
\text { AXB }_{m}\end{array}$ & $\mathrm{AXB}_{\mathrm{w}, \mathrm{FFF}}$ & $\begin{array}{l}\text { AAA vs. } \\
\text { AXB }_{\mathrm{w}, \mathrm{FFF}}\end{array}$ & $\mathrm{AXB}_{\mathrm{w}}$ & $\begin{array}{c}\text { AAA vs. } \\
\text { AXB }_{w}\end{array}$ \\
\hline PTV70 & Mean \pm SD & Mean \pm SD & p-value & Mean \pm SD & p-value & Mean \pm SD & p-value & Mean \pm SD & p-value \\
\hline D98 (Gy) & $\begin{array}{c}67.45 \pm \\
0.86\end{array}$ & $\begin{array}{c}65.17 \pm \\
1.47\end{array}$ & $<0.001$ & $\begin{array}{c}65.82 \pm \\
1.49\end{array}$ & $<0.001$ & $\begin{array}{c}66.05 \pm \\
1.05\end{array}$ & $<0.001$ & $\begin{array}{c}66.72 \pm \\
1.06\end{array}$ & $<0.001$ \\
\hline D95 (Gy) & $\begin{array}{c}68.23 \pm \\
0.67\end{array}$ & $\begin{array}{c}66.14 \pm \\
1.01\end{array}$ & $<0.001$ & $\begin{array}{c}66.80 \pm \\
1.02\end{array}$ & $<0.001$ & $\begin{array}{c}66.87 \pm \\
0.84\end{array}$ & $<0.001$ & $\begin{array}{c}67.54 \pm \\
0.85\end{array}$ & $<0.001$ \\
\hline D50 (Gy) & $\begin{array}{c}70.96 \pm \\
0.61\end{array}$ & $\begin{array}{c}68.91 \pm \\
0.84\end{array}$ & $<0.001$ & $\begin{array}{c}69.60 \pm \\
0.85\end{array}$ & $<0.001$ & $\begin{array}{c}69.68 \pm \\
0.72\end{array}$ & $<0.001$ & $\begin{array}{c}70.38 \pm \\
0.72\end{array}$ & $<0.001$ \\
\hline D2 (Gy) & $\begin{array}{c}73.12 \pm \\
0.76\end{array}$ & $\begin{array}{c}71.72 \pm \\
0.84\end{array}$ & $<0.001$ & $\begin{array}{c}72.44 \pm \\
0.85\end{array}$ & $<0.001$ & $\begin{array}{c}72.61 \pm \\
0.73\end{array}$ & 0.003 & $\begin{array}{c}73.34 \pm \\
0.74\end{array}$ & 0.156 \\
\hline $\mathrm{HI}$ & $\begin{array}{c}0.080 \pm \\
0.017\end{array}$ & $\begin{array}{l}0.10 \pm \\
0.021\end{array}$ & $<0.001$ & $\begin{array}{c}0.095 \pm \\
0.021\end{array}$ & $<0.001$ & $\begin{array}{l}0.09 \pm \\
0.018\end{array}$ & $<0.001$ & $\begin{array}{c}0.094 \pm \\
0.018\end{array}$ & $<0.001$ \\
\hline PTV70_bone & Mean \pm SD & Mean \pm SD & p-value & Mean \pm SD & p-value & Mean \pm SD & p-value & Mean \pm SD & p-value \\
\hline D98 (Gy) & $\begin{array}{c}67.33 \pm \\
1.23\end{array}$ & $\begin{array}{c}64.51 \pm \\
1.21\end{array}$ & $<0.001$ & $\begin{array}{c}65.16 \pm \\
1.23\end{array}$ & $<0.001$ & $\begin{array}{c}67.53 \pm \\
1.00\end{array}$ & 0.369 & $\begin{array}{c}68.21 \pm \\
1.01\end{array}$ & $<0.001$ \\
\hline D95 (Gy) & $\begin{array}{c}68.12 \pm \\
1.05\end{array}$ & $\begin{array}{c}65.28 \pm \\
1.06\end{array}$ & $<0.001$ & $\begin{array}{c}65.93 \pm \\
1.07\end{array}$ & $<0.001$ & $\begin{array}{c}68.19 \pm \\
0.91\end{array}$ & 0.728 & $\begin{array}{c}68.87 \pm \\
0.92\end{array}$ & $<0.001$ \\
\hline D50 (Gy) & $\begin{array}{c}70.63 \pm \\
0.80\end{array}$ & $\begin{array}{c}67.73 \pm \\
0.79\end{array}$ & $<0.001$ & $\begin{array}{c}68.41 \pm \\
0.80\end{array}$ & $<0.001$ & $\begin{array}{c}70.52 \pm \\
0.74\end{array}$ & 0.597 & $\begin{array}{c}71.23 \pm \\
0.75\end{array}$ & 0.008 \\
\hline D2 (Gy) & $\begin{array}{c}73.06 \pm \\
0.97\end{array}$ & $\begin{array}{c}70.70 \pm \\
0.88\end{array}$ & $<0.001$ & $\begin{array}{c}71.40 \pm \\
0.89\end{array}$ & $<0.001$ & $\begin{array}{c}74.23 \pm \\
1.10\end{array}$ & $<0.001$ & $\begin{array}{c}74.98 \pm \\
1.11\end{array}$ & $<0.001$ \\
\hline $\mathrm{HI}$ & $\begin{array}{c}0.081 \pm \\
0.02\end{array}$ & $0.09 \pm 0.03$ & $<0.001$ & $\begin{array}{c}0.091 \pm \\
0.03\end{array}$ & $<0.001$ & $0.10 \pm 0.02$ & $<0.001$ & $\begin{array}{c}0.095 \pm \\
0.02\end{array}$ & $<0.001$ \\
\hline PTV58.1 & Mean \pm SD & Mean \pm SD & p-value & Mean \pm SD & p-value & Mean \pm SD & p-value & Mean \pm SD & p-value \\
\hline D98 (Gy) & $\begin{array}{c}55.03 \pm \\
1.03\end{array}$ & $\begin{array}{c}53.56 \pm \\
1.12 \\
\end{array}$ & $<0.001$ & $\begin{array}{c}54.10 \pm \\
1.13\end{array}$ & $<0.001$ & $\begin{array}{c}53.90 \pm \\
0.98\end{array}$ & $<0.001$ & $\begin{array}{c}54.44 \pm \\
0.99\end{array}$ & $<0.001$ \\
\hline D95 (Gy) & $\begin{array}{c}56.40 \pm \\
0.79\end{array}$ & $\begin{array}{c}54.80 \pm \\
0.97\end{array}$ & $<0.001$ & $\begin{array}{c}55.35 \pm \\
0.98\end{array}$ & $<0.001$ & $\begin{array}{c}55.12 \pm \\
0.84\end{array}$ & $<0.001$ & $\begin{array}{l}55.67 \pm \\
0.85\end{array}$ & $<0.001$ \\
\hline D50 (Gy) & $\begin{array}{c}60.48 \pm \\
0.99\end{array}$ & $\begin{array}{c}58.81 \pm \\
1.20\end{array}$ & $<0.001$ & $\begin{array}{c}59.40 \pm \\
1.22\end{array}$ & $<0.001$ & $\begin{array}{c}59.04 \pm \\
1.10\end{array}$ & $<0.001$ & $\begin{array}{c}59.63 \pm \\
1.11\end{array}$ & $<0.001$ \\
\hline D2 (Gy) & $\begin{array}{c}69.57 \pm \\
2.61\end{array}$ & $\begin{array}{c}67.47 \pm \\
2.72\end{array}$ & $<0.001$ & $\begin{array}{c}68.14 \pm \\
2.75\end{array}$ & $<0.001$ & $\begin{array}{c}68.11 \pm \\
2.75\end{array}$ & $<0.001$ & $\begin{array}{c}68.79 \pm \\
2.78\end{array}$ & 0.014 \\
\hline
\end{tabular}

were calculated by AAA (Fig. 1D). Similarly, dosevolume parameters in PTV70_bone were partially compensated for the errors in the FFF beam, resulting in negligible dose differences in D98, D95 and D50 between AAA and $\mathrm{AXB}_{\mathrm{w}, \mathrm{FFF}}$ and mean dose difference of $1.6 \%$ for D2 (Fig. 1D). By contrast, the reduction of doses predicted by $\mathrm{AXB}_{\mathrm{m}}$ to PTV70_bone was relatively increased by the effect of the errors in the FFF beam, resulting in $\mathrm{AXB}_{\mathrm{m}, \mathrm{FFF}}$ mean dose difference from AAA of approximately $-4 \%$ for D98, D95 and D50 (Fig. 1C), with the maximum dose difference being D95 to PTV70_bone $(-6.3 \%)$.

\section{D95 dose-volume parameter in AAA versus $A X B$ modes}

The D95 dose-volume parameter is especially relevant with respect to the minimum PTV coverage that is required for the approval of a dosimetric plan. In our service, the requirement of D95 > 95\% of the prescribed dose must be satisfied for a dosimetric plan to be approved by the physician. Figure 1 shows that AAA overestimated D95 for PTV70 and PTV58.1 compared to any AXB mode, although the highest dose overestimation occurred when switching from AAA to $\mathrm{AXB}_{\mathrm{m}, \mathrm{FFF}}$ (Fig. 1C). The lowest average dose differences for D95 in 
A

$\mathrm{AXB}_{\mathrm{m}}$ mean dose difference from AAA (\%): $\left(A X B_{m}-A A A\right) / A A A$

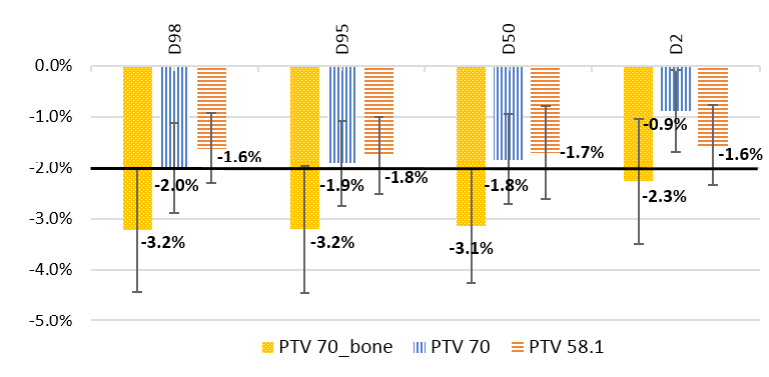

C

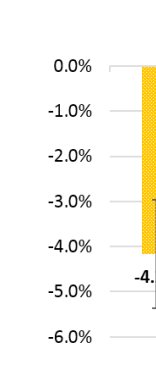

$A X B_{m, F F F}$ mean dose difference from $A A A(\%)$ : $\left(A X B_{m, F F F}-A A A\right) / A A A$

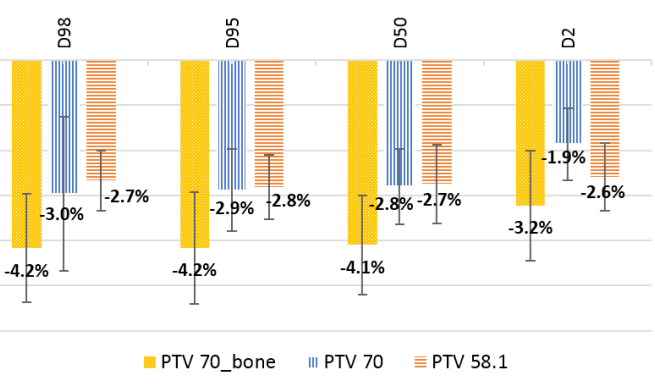

\section{B}

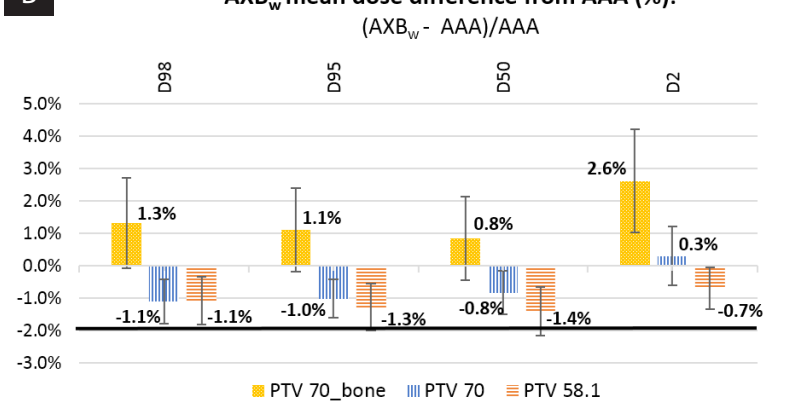

D

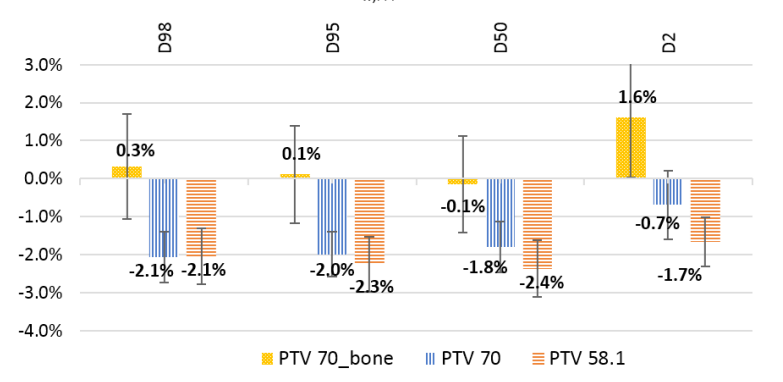

Figure 1. PTV70, PTV70 bone and PTV58.1 mean values and standard deviations of the relative dose differences for (A) AAA vs.

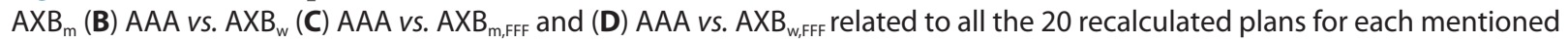
structure. The error bars represent one standard deviation. The black lines in figures (A) and (B) represent the $2 \%$ threshold value for the maximum recommended uncertainty of the computed dose distributions [36]

PTV70 and PTV58.1 were identified in the AAA vs. $\mathrm{AXB}_{\mathrm{w}}$ comparison (Fig. 1B).

To investigate the clinical impact of our findings for D95, p-values for the correlation between the D95 > 95\% requirement in PTV70 and occurrence of local recurrence for a given calculation mode $\left(\mathrm{AXB}_{\mathrm{m}}, \mathrm{AXB}_{\mathrm{m}, \mathrm{FFF}}, \mathrm{AXB}_{\mathrm{w}}\right.$ or $\left.\mathrm{AXB}_{\mathrm{w}, \mathrm{FFF}}\right)$ were computed. As shown in Supplementary Table 1, none of the

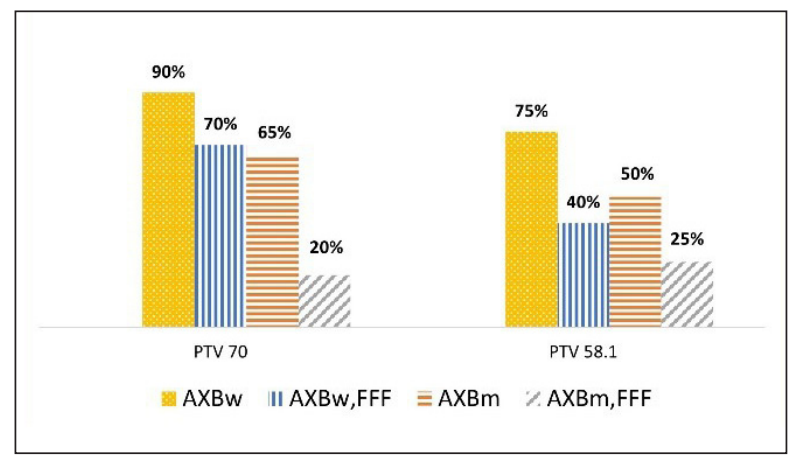

Figure 2. Percentages of patients whose dosimetric plans satisfied the requirement of D95 $>95 \%$ of the prescribed dose for each recalculated Acuros XB algorithm (AXB) plan with fixed monitor units (MU) $\mathrm{p}$-values were statistically significant $(\mathrm{p}>0.05$ in all cases), indicating that no correlation was found between these parameters.

Figure 2 supplements this information by plotting the percentages of patients whose dosimetric plans satisfied the D95 > 95\% requirement for each recalculated AXB plan with fixed MU. The dose reduction in PTV coverage, which was reflected in lower D95 values for any AXB calculation compared to AAA, resulted in a significant decrease in the percentage of patients whose dosimetric plans would have satisfied the D95 > 95\% requirement, especially for the AXB calculations (Fig. 2). The most important reduction occurred for the $\mathrm{AXB}_{\mathrm{m}, \mathrm{FFF}}$ calculation, for which only $20 \%$ and $25 \%$ of patient plans would have been approved for PTV70 and PTV58.1, respectively. However, despite this striking result, it is important to note that the D95 > 95\% constraint does not imply a strict dichotomic criterion. Other clinical factors may influence the approval or rejection of a given dosimetric plan, and the percentage of the target volume to be covered by the D95 isodosis may vary from centre to centre. 


\section{Discussion}

The AAA algorithm predicts uncertainties in the dose calculation for various sites that result in approximately $1-2 \%$ underdosage of the tumour [11, 37]. In particular, an overall typical $1 \%$ underdosage in tissue and up to approximately $5 \%$ dose reduction in bone have been reported for $\mathrm{H} \& \mathrm{~N}$ cancer when comparing the AXB versus the AAA algorithm [24-26, 38]. However, the extent to which the additional uncertainty that is introduced by using unfiltered beams could lead to significant underdosage of the tumour has not yet been determined. In the current study, we incorporated the systematic error in the FFF beam calibration when using the AXB algorithm in our head and neck FFF-VMAT plans, demonstrating that if we use the AAA algorithm and conduct an absolute dose calibration of the FFF beam according to the TRS-398 protocol, we might, in the worst-case scenario, underdose the tumour by up to $4.1 \%$. Additionally, if we consider the bony structures within the tumour, the underdosage within these areas might reach up to $6.3 \%$ in the worst-case scenario.

Regarding the dosimetric impact of switching from $\mathrm{AAA}$ to $\mathrm{AXB}$, our results, which are in agreement with the literature $[11,18,27,28,39]$, show that the AAA algorithm systematically overestimated the target dose compared to AXB. Several studies [27, 28, 40] have examined the mean dose differences in PTV dose-volume parameters, such as D98, D95, D50 and D2, when comparing AAA against AXB in H\&N treatments, and their results in terms of systematic differences were similar to those that were obtained in our work. AAA overestimated all these dose-volume parameters compared to AXB, except D2 in PTV70, and all dose-volume parameters in PTV70_bone which were systematically higher when computed with $\mathrm{AXB}_{w}$ (Fig. 1B). This result in D2 and dosimetric parameters in PTV70_bone have been previously explained in terms of the presence of high- $\mathrm{Z}$ materials (bone or cartilage) in PTV $[25,27,28]$. In addition, the random presence of these high- $\mathrm{Z}$ materials in PTV and the resulting "hot spots" can lead to differences in the widths of the dose distributions of the dose-volume parameters thereby giving rise to nonstatistically significant $\mathrm{p}$-values, such as p-values for D2 and dosimetric parameters in PTV70 in the comparison between $\mathrm{AAA}$ and $\mathrm{AXB}_{\mathrm{w}}$ that is presented in Table 2.

In addition, our results show that the dose overestimation was more pronounced if we compared AAA with $\mathrm{AXB}_{\mathrm{m}}$ than if we compared AAA with $\mathrm{AXB}_{w}$ with mean dose differences in both cases of less than 2\% (Fig. 1A,B). This is in agreement with the findings of Zifodya et al. [27] who reported a PTV dose that was lower by approximately $1.5 \%$ when calculated with AAA. Muñoz et al. [28] also came to a similar conclusion with a dose reduction of approximately $1.7 \%$ when using AAA. For the homogeneity index (HI), in Table 2, the lower HI values for AAA compared to those for AXB modes indicate that AAA produced more homogeneous plans, which is in agreement with the two abovementioned studies that also assessed differences in HI between AAA and AXB in H\&N treatments [27, 28]. In addition, the significant differences in the $\mathrm{HI}$ values for the comparisons of AAA with $\mathrm{AXB}_{\mathrm{m}}$ and $\mathrm{AXB}_{w}$, as presented in Table 2 , are also in agreement with the findings of Zifodya et al. [27], who explain that these differences are due to the superior performance of AXB in estimating doses for the heterogeneous media in the $\mathrm{H} \& \mathrm{~N}$ site.

In summary, our findings on target dose reduction when AAA is used in $\mathrm{H} \& \mathrm{~N}$ treatments agree with published results; however, it is not clear whether there are published data on the added target dose reduction when a non-rigorous dose calibration of the unfiltered beam is conducted. Although two previous studies [41, 42] assessed the dose differences between AAA and AXB in VMAT treatments in heterogeneous media with FFF beams, they did not specify the inclusion of additional corrections for an accurate dose calibration of FFF beams. Our novel approach showed that when these corrections were included, the comparison of AAA with AXB yielded dose differences of up to $4.1 \%$ in PTV70 D98 and $6.3 \%$ in $\mathrm{PTV} 70_{\text {bone }} \mathrm{D} 95$. This finding regarding the impact of using FFF beams in VMAT treatments is interesting, as it differs from the results of the two previously discussed works [41, 42], which reported dose differences between AAA and AXB of less than $2 \%$. Muralidhar et al. [41] found maximum dose differences between AAA and AXB of 1.7\% for the 6XFFF beam in heterogeneous phantoms for lung SBRT treatments, while Huang et al. [42] reported a mean dose difference between AAA 
and $\mathrm{AXB}$ of $1.33 \pm 1.12 \%$ for the $6 \mathrm{XFFF}$ beam in lung cancer patients who were treated via the same technique. It is likely that these authors did not conduct a robust FFF beam dose calibration such as that conducted in our work as they did not discuss it and it is not addressed in international dosimetry protocols. The different sites and radiotherapy techniques that were used in their work (lung SBRT VMAT) could also influence the dose differences between the two algorithms. It has been reported that the differences between doses that were calculated by AAA and AXB in heterogeneous media may be influenced by factors that are associated with the treatment technique (such as the beam energy, the field size, and the number of fields) and the site (such as the density of the medium) [10]. Therefore, further investigation is necessary for determining the reasons for this inconsistency.

With respect to the measurements in FFF beams, the results of our study are consistent with those of several authors [32-35] who investigated the influence of the uncorrected $\operatorname{PDD}(10)$ on the absolute dose determination in FFF beams. Particularly, only Sudhyadhom et al. [32] evaluated the impact of the overall systematic error that was due to volume-averaging and ion recombination effects in $\mathrm{PDD}(10)$ on the absolute dose calibration of the FFF beam using the chambers that were used in our work. They found errors of $1.6 \%$ and $1 \%$ for $10 \mathrm{XFFF}$ and $6 \mathrm{XFFF}$, respectively, which accord with our reported value of $1 \%$ for the $7 \mathrm{XFFF}$ beam.
In conclusion, the overall dosimetric impact of switching from AAA to AXB in our H\&N FFFVMAT treatments was significant. The reduction in the dose to $95 \%$ of both PTVs led to a considerable reduction in the percentage of approved patient plans after recalculation with AXB of up to $20 \%$ in the worst-case scenario. Figure 3 illustrates an example of a representative patient, which clearly shows a significant reduction in the tumour coverage by the $95 \%$ prescription dose when switching from $A A A$ to $\mathrm{AXB}_{\mathrm{m}, \mathrm{FFF}}$. This significant reduction in the tumour dose coverage increases the likelihood of local recurrence. However, despite these striking outcomes, there seems to be no clinical evidence of local recurrence that resulted from such underdosage of the tumour in treatments of this type in the literature, nor has this been observed in our study (none of the p-values for the correlation between the D95 > 95\% requirement and occurrence of local recurrences were statistically significant, as shown in Supplementary Table 1). Most of the current clinical experience is based on AAA or similar algorithms and on calibration protocols and clinical trials that are dose-to-water-based. We suspect that this is the main reason for the lack of clinical impact of these deficiencies in the dose coverage that are predicted by the AXB algorithm. Nevertheless, the key role that accurate dose reporting plays in clinical trials to improve their robustness must be considered. In this regard, both the dose reporting mode and FFF beam calibration procedure must be carefully reviewed to ensure maximum dose accuracy.

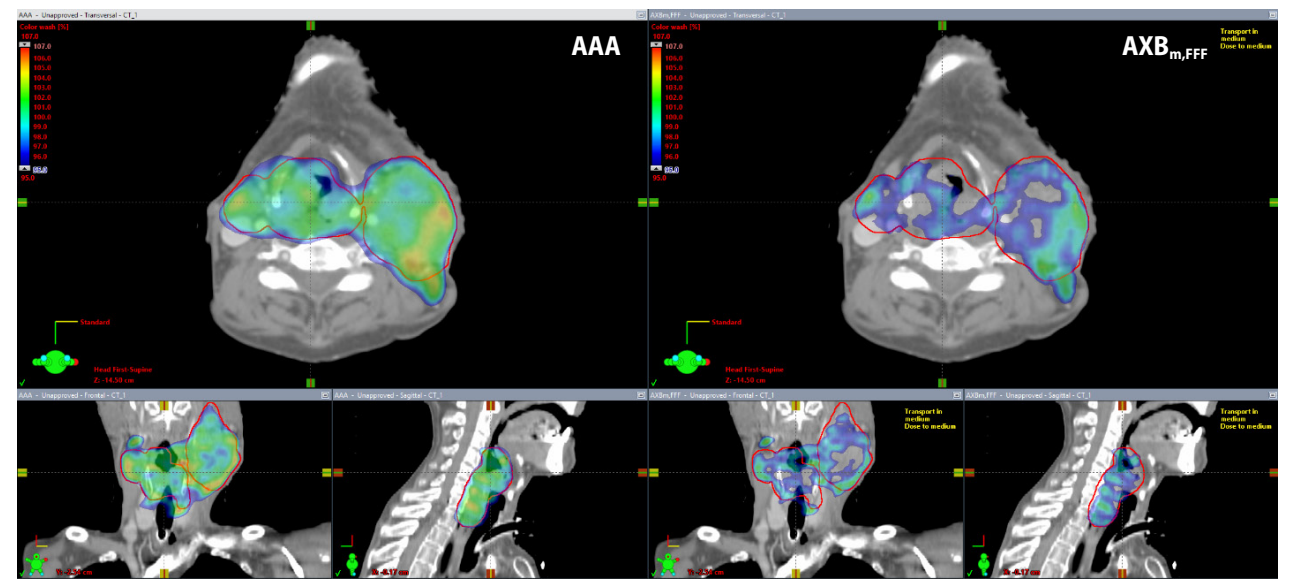

Figure 3. Tumour contours (PTV70) which are represented by red lines, and dose distributions which are painted by colour wash from $95 \%$ to $107 \%$ isodose lines. Axial, frontal and sagittal views for the 7XFFF VMAT plans that were calculated for the same MU using a) AAA and b) $A X B_{m, F F}$ 
To the best of our knowledge, only one study has been published that links switching from AAA to $\mathrm{AXB}_{\mathrm{m}}$ with the clinical outcomes of the $\mathrm{H} \& \mathrm{~N}$ patients. In this recently published study, Hardcastle et al. [38] showed that although patients were treated with lower than appreciated doses at the time of plan acceptance, they were all cured locoregionally. They conclude that for their patient cohort there is no basis for accepting higher doses with new planning algorithms since the chances of long-term harm to healthy tissue will increase without a clear benefit in terms of the tumour control probability. However, notwithstanding this important conclusion, their study of selected patients with a specified type of $H \& N$ cancer does not resolve the clinical conundrum of whether to switch from AAA to $\mathrm{AXB}_{\mathrm{m}}$ widely in $\mathrm{H} \& \mathrm{~N}$ patients. The decision to adopt this change, which involves a dose increase without comparative clinical data, is difficult to take. In the case of our study, in which a significant underdosage of the tumour was observed, the decision was clear: switch to the $\mathrm{AXB}_{\mathrm{m}}$ algorithm and conduct a more robust calibration of the FFF beam due to the likelihood of tumour control probability loss. The main reasons for choosing $\mathrm{AXB}_{\mathrm{m}}$ instead of $\mathrm{AXB}_{\mathrm{w}}$ were its higher accuracy and consistency. Although the objective of this study is not to resolve the current debate on which AXB mode should be used [21, 43, 44], we agree with Reynaert et al. [45] that it is fundamental to have a consensus on how to report doses to improve the consistency among future data. In this regard, the NRG has recommended the use of dose-to-medium values for dose specification for all its clinical trials [46]. Their main justifications for this recommendation were that the use of dose-to-medium values provides higher accuracy and follows the current trend in algorithm development, namely, increasingly many algorithms are becoming available that can calculate the dose-to-medium values. Furthermore, according to the report of the AAPM Task Group 329 on reference dose specification for dose calculations [47], the use of the Acuros algorithm's dose-towater reporting mode is not recommended. In this context, Andreo [44] conducted an in-depth analysis on the dosimetric impact of converting dose to tissue into dose to water-equivalent tissue, assuming that all body tissues are water-like. His study showed that a retrospective conversion from dose-to-tissue to dose-to-water, based on a widely used approach, would mostly increase the final uncertainty of the treatment planning process. Therefore, he concluded that planning in terms of dose-to-tissue should be the preferred choice, always keeping in mind the approximations included in numerical transport methods and the difference obtained with various TPS for bone and adipose tissues.

Finally, our future work will focus on the evaluation of dose-volume parameters of OAR in H\&N VMAT treatments with FFF beams and their clinical impact.

\section{Conclusions}

This study shows that dose differences between the AAA and AXB algorithms can be as large as $6.3 \%$ in H\&N FFF-VMAT treatments if the unfiltered beam is not accurately characterized. This led to a significant at-risk nodal region and tumour underdosage, particularly for bony structures within the tumour, when using $\mathrm{AXB}_{\mathrm{m}}$ calculations. However, while this underdosing in targets does not seem to have major clinical consequences, it is essential to report the dose as accurately as possible to improve the robustness of clinical trials. In this context, further research is needed on the clinical impact of dose reductions on tumour and healthy tissue that are predicted by the $\mathrm{AXB}_{\mathrm{m}}$ algorithm, as most current clinical experience is based on AAA algorithms and dose-to-water dosimetry.

In conclusion, based on the results of this work, the authors of this study recommend switching to the $\mathrm{AXB}_{\mathrm{m}}$ algorithm and carefully reviewing the FFF beam dose calibration procedure in $\mathrm{H} \& \mathrm{~N}$ FFF-VMAT treatments. This novel approach will ensure (1) an increase in dose calculation accuracy by approximately $3 \%$; (2) a consequent dose increase in targets; and (3) a dose reporting mode that is consistent with the trend of current algorithms.

\section{Conflicts of interest}

The authors have no relevant conflicts of interest to disclose.

\section{Financial disclosure}

There are no financial conflicts of interest to disclose. 


\section{Acknowledgments}

The authors would like to offer their special thanks to Claudio Fiorino for his sharp and valuable comments. We also sincerely thank Rocío Bermudez Luna for providing initial patient data, which were very helpful for this investigation.

\section{References}

1. Xiao Y, Kry SF, Popple R, et al. Flattening filter-free accelerators: a report from the AAPM Therapy Emerging Technology Assessment Work Group. J Appl Clin Med Phys. 2015; 16(3): 5219, doi: 10.1120/jacmp.v16i3.5219, indexed in Pubmed: 26103482.

2. O'Brien PF, Gillies BA, Schwartz M, et al. Radiosurgery with unflattened 6-MV photon beams. Med Phys. 1991; 18(3): 519-521, doi: 10.1118/1.596656, indexed in Pubmed: 1908047.

3. Vassiliev ON, Titt U, Pönisch F, et al. Dosimetric properties of photon beams from a flattening filter free clinical accelerator. Phys Med Biol. 2006; 51(7): 19071917, doi: 10.1088/0031-9155/51/7/019, indexed in Pubmed: 16552113.

4. Cashmore J.The characterization of unflattened photon beams from a 6 MV linear accelerator. Phys Med Biol. 2008; 53(7): 1933-1946, doi: 10.1088/0031-9155/53/7/009, indexed in Pubmed: 18364548.

5. Fogliata A, Vanetti E, Albers D, et al. On the dosimetric behaviour of photon dose calculation algorithms in the presence of simple geometric heterogeneities: comparison with Monte Carlo calculations. Phys Med Biol. 2007; 52(5): 1363-1385, doi: 10.1088/0031-9155/52/5/011, indexed in Pubmed: 17301460.

6. Chopra K, Leo P, Kabat C, et al. Evaluation of dose calculation accuracy of treatment planning systems in the presence of tissue heterogeneities. Ther Radiol Oncol. 2018; 2: 28-28, doi: 10.21037/tro.2018.07.01.

7. Knöös T, Wieslander E, Cozzi L, et al. Comparison of dose calculation algorithms for treatment planning in external photon beam therapy for clinical situations. Phys Med Biol. 2006; 51(22): 5785-5807, doi: 10.1088/00319155/51/22/005, indexed in Pubmed: 17068365.

8. Ojala JJ, Kapanen MK, Hyödynmaa SJ, et al. Performance of dose calculation algorithms from three generations in lung SBRT: comparison with full Monte Carlo-based dose distributions. J Appl Clin Med Phys. 2014; 15(2): 4662, doi: $10.1120 /$ jacmp.v15i2.4662, indexed in Pubmed: 24710454.

9. Kisling KD, Ger RB, Netherton TJ, et al. A snapshot of medical physics practice patterns. J Appl Clin Med Phys. 2018; 19(6): 306-315, doi: 10.1002/acm2.12464, indexed in Pubmed: 30272385.

10. Fogliata A, Nicolini G, Clivio A, et al. Dosimetric evaluation of Acuros XB Advanced Dose Calculation algorithm in heterogeneous media. Radiat Oncol. 2011; 6: 82, doi: 10.1186/1748-717X-6-82, indexed in Pubmed: 21771317.

11. Fogliata A, Nicolini G, Clivio A, et al. Critical appraisal of Acuros XB and Anisotropic Analytic Algorithm dose calculation in advanced non-small-cell lung cancer treatments. Int J Radiat Oncol Biol Phys. 2012; 83(5):
1587-1595, doi: 10.1016/j.ijrobp.2011.10.078, indexed in Pubmed: 22300575.

12. Kathirvel M, Subramanian S, Clivio A, et al. Critical appraisal of the accuracy of Acuros-XB and Anisotropic Analytical Algorithm compared to measurement and calculations with the compass system in the delivery of RapidArc clinical plans. Radiat Oncol. 2013; 8: 140, doi: 10.1186/1748717X-8-140, indexed in Pubmed: 23758728.

13. Ono K, Endo S, Tanaka K, et al. Dosimetric verification of the anisotropic analytical algorithm in lung equivalent heterogeneities with and without bone equivalent heterogeneities. Med Phys. 2010; 37(8): 4456-4463, doi: 10.1118/1.3464748, indexed in Pubmed: 20879604.

14. Robinson D. Inhomogeneity correction and the analytic anisotropic algorithm. J Appl Clin Med Phys. 2008; 9(2): 112-122, doi: 10.1120/jacmp.v9i2.2786, indexed in Pubmed: 18714283.

15. Vassiliev ON, Wareing TA, McGhee J, et al. Validation of a new grid-based Boltzmann equation solver for dose calculation in radiotherapy with photon beams. Phys Med Biol. 2010; 55(3): 581-598, doi: 10.1088/0031-9155/55/3/002, indexed in Pubmed: 20057008.

16. Vassiliev ON, Wareing TA, Davis IM, et al. Feasibility of a multigroup deterministic solution method for threedimensional radiotherapy dose calculations. Int J Radiat Oncol Biol Phys. 2008; 72(1): 220-227, doi: 10.1016/j. ijrobp.2008.04.057, indexed in Pubmed: 18722273.

17. Failla GA, Wareing T, Archambault Y, Thompson S. Acuros ${ }^{\circledR}$ $\mathrm{XB}$ avanced dose calculation for the EclipseTM treatment planning system n.d: 14. 2010.

18. Kroon PS, Hol S, Essers M. Dosimetric accuracy and clinical quality of Acuros XB and AAA dose calculation algorithm for stereotactic and conventional lung volumetric modulated arc therapy plans. Radiat Oncol. 2013; 8: 149, doi: 10.1186/1748-717X-8-149, indexed in Pubmed: 23800024.

19. Bush K, Gagne IM, Zavgorodni S, et al. Dosimetric validation of Acuros XB with Monte Carlo methods for photon dose calculations. Med Phys. 2011; 38(4): 2208-2221, doi: 10.1118/1.3567146, indexed in Pubmed: 21626955.

20. Han T, Mikell JK, Salehpour M, et al. Dosimetric comparison of Acuros XB deterministic radiation transport method with Monte Carlo and model-based convolution methods in heterogeneous media. Med Phys. 2011; 38(5): 2651-2664, doi: 10.1118/1.3582690, indexed in Pubmed: 21776802.

21. Chetty IJ, Curran B, Cygler JE, et al. Report of the AAPM Task Group No. 105: Issues associated with clinical implementation of Monte Carlo-based photon and electron external beam treatment planning. Med Phys. 2007; 34(12): 4818-4853, doi: 10.1118/1.2795842, indexed in Pubmed: 18196810.

22. Han T, Mourtada F, Kisling K, et al. Experimental validation of deterministic Acuros XB algorithm for IMRT and VMAT dose calculations with the Radiological Physics Center's head and neck phantom. Med Phys. 2012; 39(4): 2193-2202, doi: 10.1118/1.3692180, indexed in Pubmed: 22482641.

23. Kan MWK, Leung LHT, Yu PKN. Verification and dosimetric impact of Acuros XB algorithm on intensity modulated stereotactic radiotherapy for locally persistent nasopharyngeal carcinoma. Med Phys. 2012; 39(8): 4705-4714, doi: 10.1118/1.4736819, indexed in Pubmed: 22894395. 
24. Kan MWK, Leung LHT, So RWK, et al. Experimental verification of the Acuros $\mathrm{XB}$ and $\mathrm{AAA}$ dose calculation adjacent to heterogeneous media for IMRT and RapidArc of nasopharygeal carcinoma. Med Phys. 2013; 40(3): 031714, doi: 10.1118/1.4792308, indexed in Pubmed: 23464309.

25. Kan MWK, Leung LHT, Yu PKN. Dosimetric impact of using the Acuros XB algorithm for intensity modulated radiation therapy and RapidArc planning in nasopharyngeal carcinomas. Int J Radiat Oncol Biol Phys. 2013; 85(1): e73-e80, doi: 10.1016/j.ijrobp.2012.08.031, indexed in Pubmed: 23040220.

26. Hirata K, Nakamura M, Yoshimura M, et al. Dosimetric evaluation of the Acuros $\mathrm{XB}$ algorithm for a $4 \mathrm{MV}$ photon beam in head and neck intensity-modulated radiation therapy. J Appl Clin Med Phys. 2015; 16(4): 52-64, doi: 10.1120/ jacmp.v16i4.5222, indexed in Pubmed: 26218997.

27. Zifodya JM, Challens CHC, Hsieh WL. From AAA to Acuros $\mathrm{XB}$-clinical implications of selecting either Acuros XB dose-to-water or dose-to-medium. Australas Phys Eng Sci Med. 2016; 39(2): 431-439, doi: 10.1007/s13246-0160436-z, indexed in Pubmed: 27008647.

28. Muñoz-Montplet C, Marruecos J, Buxó M, et al. Dosimetric impact of Acuros XB dose-to-water and dose-to-medium reporting modes on VMAT planning for head and neck cancer. Phys Med. 2018; 55: 107-115, doi: 10.1016/j. ejmp.2018.10.024, indexed in Pubmed: 30471814.

29. Yeh P, Lee CC, Chao TC, et al. Monte Carlo evaluation of Acuros XB dose calculation Algorithm for intensity modulated radiation therapy of nasopharyngeal carcinoma. Radiat Phys Chem. 2017; 140: 419-422, doi: 10.1016/j. radphyschem.2017.02.025.

30. Anreo P, Burns DT, Hohlfel K, et al. Absorbed Dose Determination in External Beam Radiotherapy: An International Code of Practice for Dosimetry based on Standards of Absorbed Dose to Water. International Atomic Energy Agency, Vienna 2006.

31. McEwen M, DeWerd L, Ibbott G, et al. Addendum to the AAPM's TG-51 protocol for clinical reference dosimetry of high-energy photon beams. Med Phys. 2014; 41(4): 041501-24, doi: 10.1118/1.4866223, indexed in Pubmed: 24694120.

32. Sudhyadhom A, Kirby N, Faddegon B, et al. Technical Note: Preferred dosimeter size and associated correction factors in commissioning high dose per pulse, flattening filter free x-ray beams. Med Phys. 2016; 43(3): 1507-1513, doi: 10.1118/1.4941691, indexed in Pubmed: 26936734.

33. Corns RA, Huang VW, Thomas SD. Pion effects in flattening filter-free radiation beams. J Appl Clin Med Phys. 2015; 16(6): 376-385, doi: 10.1120/jacmp.v16i6.5869, indexed in Pubmed: 26699592.

34. Sutton J, Littler J. Accounting for the ion recombination factor in relative dosimetry of flattening filter free photon radiation. Biomed Phys Engineer Express. 2017; 3(1): 017002, doi: 10.1088/2057-1976/aa5493.

35. Vargas Castrillón S, Cutanda Henríquez F. Choice of a Suitable Dosimeter for Photon Percentage Depth Dose Measurements in Flattening Filter-Free Beams. J Med Phys. 2017;42(3): 140-143, doi: 10.4103/jmp.JMP_11_17, indexed in Pubmed: 28974859.

36. AAPM Reports - Tissue Inhomogeneity Corrections for Megavoltage Photon Beams . https://www.aapm.org/ pubs/reports/detail.asp?docid=86 (May 13, 2019).
37. Fogliata A, Nicolini G, Clivio A, et al. On the dosimetric impact of inhomogeneity management in the Acuros XB algorithm for breast treatment. Radiat Oncol. 2011; 6: 103, doi: 10.1186/1748-717X-6-103, indexed in Pubmed: 21871079.

38. Hardcastle N, Montaseri A, Lydon J, et al. Dose to medium in head and neck radiotherapy: Clinical implications for target volume metrics. Phys Imag Radiat Oncol. 2019; 11: 92-97, doi: 10.1016/j.phro.2019.08.005.

39. Fogliata A, Scorsetti M, Navarria P, et al. Dosimetric comparison between VMAT with different dose calculation algorithms and protons for soft-tissue sarcoma radiotherapy. Acta Oncol. 2013; 52(3): 545-552, doi: 10.3109/ 0284186X.2012.689853, indexed in Pubmed: 22671576.

40. Park SY, Park J, Choi C, et al. Dosimetric Validation of the Acuros XB Advanced Dose Calculation Algorithm for Volumetric Modulated Arc Therapy Plans. Progress Med Phys. 2016; 27(4): 180, doi: 10.14316/pmp.2016.27.4.180.

41. Muralidhar KR, Pangam S, Srinivas $P$, et al. A phantom study on the behavior of Acuros XB algorithm in flattening filter free photon beams. J Med Phys. 2015; 40(3): 144-149, doi: 10.4103/0971-6203.165076, indexed in Pubmed: 26500400.

42. Huang $B$, Wu L, Lin $P$, et al. Dose calculation of Acuros XB and Anisotropic Analytical Algorithm in lung stereotactic body radiotherapy treatment with flattening filter free beams and the potential role of calculation grid size. Radiat Oncol. 2015; 10: 53, doi: 10.1186/s13014-015-0357-0, indexed in Pubmed: 25886628.

43. Ma CM, Li J. Dose specification for radiation therapy: dose to water or dose to medium? Phys Med Biol. 2011;56(10): 3073-3089, doi: 10.1088/0031-9155/56/10/012, indexed in Pubmed: 21508447.

44. Andreo P. Dose to 'water-like' media or dose to tissue in MV photons radiotherapy treatment planning: still a matter of debate. Phys Med Biol. 2015; 60(1): 309-337, doi: 10.1088/0031-9155/60/1/309, indexed in Pubmed: 25503312.

45. Reynaert N, Crop F, Sterpin E, et al. On the conversion of dose to bone to dose to water in radiotherapy treatment planning systems. Phys Imag Radiat Oncol. 2018; 5: 26-30, doi: 10.1016/j.phro.2018.01.004.

46. Gladstone DJ, Kry SF, Xiao Y, et al. Dose Specification for NRG Radiation Therapy Trials. Int J Radiat Oncol Biol Phys. 2016; 95(5): 1344-1345, doi: 10.1016/j.ijrobp.2016.03.044, indexed in Pubmed: 27479721.

47. Kry SF, Feygelman V, Balter P, et al. AAPM Task Group 329: Reference dose specification for dose calculations: Dose-to-water or dose-to-muscle? Med Phys. 2020; 47(3): e52-e64, doi: 10.1002/mp.13995, indexed in Pubmed: 31883390.

48. Hyun MA, Miller JR, Micka JA, et al. Ion recombination and polarity corrections for small-volume ionization chambers in high-dose-rate, flattening-filter-free pulsed photon beams. Med Phys. 2017; 44(2): 618-627, doi: 10.1002/ mp.12053, indexed in Pubmed: 28001291.

49. Martin-Martin G, Aguilar PB, Barbés B, et al. Assessment of ion recombination correction and polarity effects for specific ionization chambers in flattening-filter-free photon beams. Phys Med. 2019; 67: 176-184, doi: 10.1016/j. ejmp.2019.07.018, indexed in Pubmed: 31734555.

50. Kry SF, Popple R, Molineu A, et al. Ion recombination correction factors (P(ion)) for Varian TrueBeam high- 
dose-rate therapy beams. J Appl Clin Med Phys. 2012; 13(6): 3803, doi: 10.1120/jacmp.v13i6.3803, indexed in Pubmed: 23149774.
51. Johnsen S. SU-GG-T-195: Ion Chamber Collection Efficiency Considerations for Un-Flattened X-Ray Beams. Med Phys. 2008; 35(6Part12): 2770-2770, doi: 10.1118/1.2961947. 